\title{
Localized Gastric Amyloidosis
}

\author{
Kazuhiro Furukawa, Ryoji Miyahara, Kohei Funasaka, Mitsuhiro Fujishiro
}

Department of Gastroenterology and Hepatology, Nagoya University Graduate School of Medicine, Nagoya, Japan

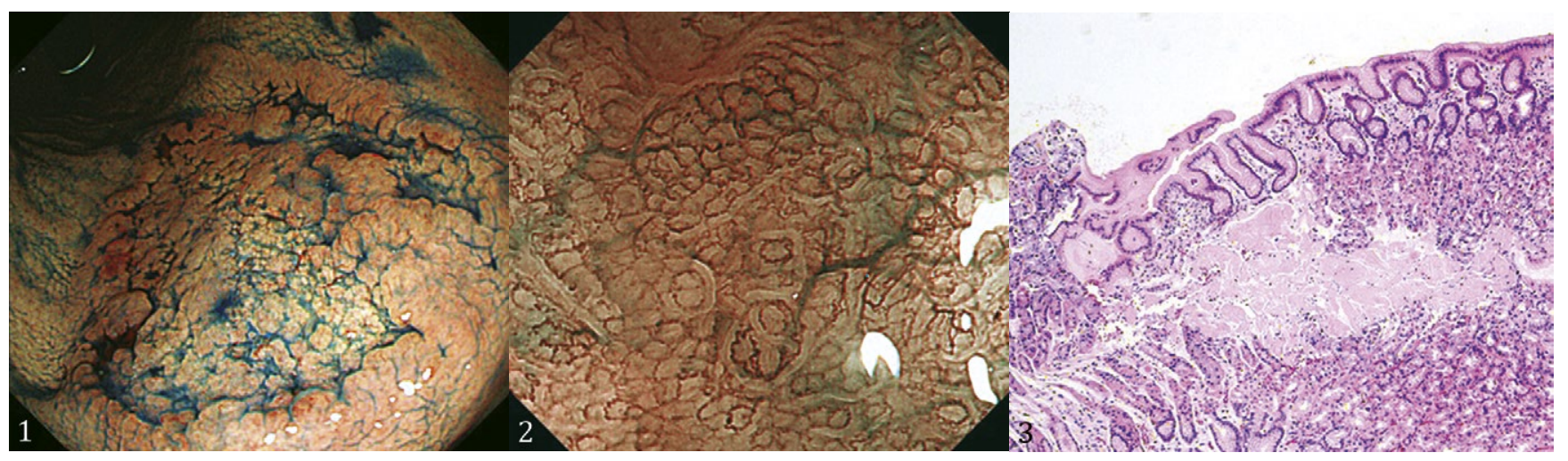

A 56-year old male was referred for a depressed lesion in his stomach. Esophagogastroduodenoscopy revealed a palecolored, slightly depressed, $4 \mathrm{~cm}$ lesion in the lesser curvature of the mid-gastric body. The lesion had a distinct demarcation line and no fold convergency (Fig. 1). Looped microvessels and dilatated vessels without variable calibers were detected on the surface by magnifying endoscopy with narrow-band imaging and there were no obvious signs of malignancy (Fig. 2). Hematoxylin-eosin staining of biopsy specimens revealed deposits of a structureless, homogeneous, and eosinophilic material in the gastric mucosa (Fig. 3). This material stained positive with direct fast scarlet, indicating that it was amyloid. On immunohistochemistry, the anti-kappa light chain antibody was positive. Amyloid deposits were not detected in other organs and multiple myeloma was excluded. Therefore, the patient was diagnosed with localized gastric amyloidosis characterized by the deposition of AL type amyloid in the mucosal layer. Further treatment was not initiated because the patient was asymptomatic. The appearance and size of gastric amyloidosis have remained unchanged in annual follow-up endoscopy for seven years.

Amyloidosis is characterized by extracellular deposition of insoluble fibrillar proteins in various organs and tissues [1]. It is classified into systemic and localized amyloidosis based on the distribution of amyloid, and is categorized by the identity of the corresponding precursor proteins. Involvement of the gastrointestinal tract is often observed in patients with systemic amyloidosis; however, the amyloid deposition confined to the stomach is extremely rare [1-3]. The gross appearance of localized gastric amyloidosis takes various forms such as depressed lesion, submucosal tumorlike or nodular appearance, and thickened folds $[1,2,4,5]$, and it is often difficult to differentiate from gastric cancer or malignant lymphoma with only conventional white light endoscopy. Magnifying endoscopy with narrow-band imaging is useful for the diagnosis of gastric amyloidosis, and a groovetype surface pattern with irregularity, round small vascular changes, and dilatated vessels without variable caliber have been reported for gastric amyloidosis $[1,4]$. The majority of patients with localized gastric amyloidosis have a favorable prognosis without morphological changes, even after longterm observations $[2,3]$.

Corresponding author: Kazuhiro Furukawa, kazufuru@med.nagoya-u.ac.jp

Conflicts of interest: None to declare.

\section{REFERENCES}

1. Kagawa M, Fujino Y, Muguruma N, et al. Localized amyloidosis of the stomach mimicking a superficial gastric cancer. Clin J Gastroenterol 2016;9:109-113. doi:10.1007/s12328-016-0651-X

2. Ahn YH, Rhee YY, Choi SC, Seo GS. Localized Gastric Amyloidosis with Kappa and Lambda Light Chain Co-Expression. Clin Endosc 2018;51:285-288. doi:10.5946/ce.2017.118

3. Wu D, Lou JY, Chen J, et al. A case report of localized gastric amyloidosis. World J Gastroenterol 2003;9:2632-2634. doi:10.3748/wjg.v9.i11.2632

4. Matsueda K, Kawano S, Okada H. Primary localized amyloidosis of the stomach mimicking healing gastric ulcer. Gastrointest Endosc 2020;91:947-948. doi:10.1016/j.gie.2019.12.004

5. Tada S, Iida M, Iwashita A, et al. Endoscopic and biopsy findings of the upper digestive tract in patients with amyloidosis. Gastrointest Endosc 1990;36:10-14. doi:10.1016/s0016-5107(90)70913-3 